\title{
Discussion on the Construction of Security System of Conference, Festival and Special Event
}

\author{
Lin Sun \\ Xiamen Huaxia University, Xiamen Fujian, 361000, China
}

Keywords: Conference, Festival and special event, Security system.

\begin{abstract}
With the flourishing of socialist market economy with Chinese characteristics, the conference and festival economy also develop in a new way. Besides, there are increasingly intensive conferences, festivals and special events. Since a conference, festival or special event usually involves abundant people, properties and materials etc., ineffective organization or supervision will probably cause a security accident, resulting in large damages to people's life and property safety. For this reason, it is very important to strengthen the construction of the security system for conference, festival and special events. This paper summarized the basic characteristics of conference, festival and special event, analyzed the major issues in the construction of security system of conference, festival and special event and emphatically elaborated the effective measures to enhance the construction of conference and festival security system.
\end{abstract}

\section{Introduction}

Nowadays, the problem of public security has become a focus problem concerned by the whole world. With the development of abundant conferences, festivals and special events of all kinds in recent years, the security problem has become a great threat to human peace and development. As the public security relates to human's life and property safety, the governments of all countries attach much importance to it. However, the existence of factors, such as rapid increase in population, frequent turnover of people and group interest differentiation caused by economic development, has resulted in that major conferences, festivals and special events become the major occasions for outburst of potential safety hazards. Compared with the western developed countries, the conference and festival economy started late in China, so the construction of security management concerned is not sound and perfect enough now. Most organizers and undertaking enterprises of a conference and festival activity lack the safety awareness in varying degrees. The construction of a scientific and standard conference and festival security system can effectively reduce and even avoid security accidents, help to control and reduce the risks of related enterprises during operation, safeguard the successful implementation of a conference and festival activity, and protect exhibitors' legal interests.

\section{Basic characteristics of conference and festival activity}

A conference, festival or special event is usually held in a large venue, the open air or a public place with high concentration of people. Because there are many people when a conference, festival or special event is held, any accident may cause a great panic among people involved; this also can easily cause a chain reaction, which may result in an accident that is unimaginable in normal conditions. The common characteristics of all sorts of conferences, festivals and special events include intensive people, suddenness, unpredictability and social influence etc.

\section{Major issues in the construction and conference and festival security system}

At present, in China's conferences, festivals and special events, the organizer and provide of conference, festival and special event are the subjects, which cooperate with the related national departments with clear duties to carry out activities. As China's legal system is continuously strengthened and perfected, the construction of conference and festival security system is also 
enhanced ceaselessly. However, it is still not perfect. There are still quite a few problems in the construction of conference and festival security system and such problems are prominently shown in following four aspects: firstly, various security emergency organizations place particular emphasis on the post treatment of an accident, don't take sufficient measures for dynamic early warning of an accident during a conference, festival or special event and lack a perfect early warning management system for security in a conference, festival and special event; secondly, the competent governmental departments concerned lack adequately smooth mutual information communications, which may result in the delay in control and response of an accident and thus lead to more property loss and even personal injury; thirdly, some organizers and undertaking enterprises of conference and festival activities as well as the conference venue providers don't make sufficient security organizations and post arrangements; in particular, they lack safety awareness, practitioner for safety management and sufficient understanding of accidents; besides, the safety precaution and emergency construction lag behind; fourthly, some organizers of conference and festival activities as well as the providers of venues for conference and festival activities don't provide their employees with sufficiently rigorous and standard education and training; some safety education and training activities carried out lack adequate pertinence, so the effect is not satisfactory.

\section{Effective measures for strengthening the construction of conference and festival security system}

\section{To strengthen publicity and education and perfect the construction of feedback system}

Since the Law of the People's Republic of China on Emergency Response was issued by our country in November 2007, the government departments at all levels has been paying high attention to the emergency management publicity and education including the publicity and education of emergency management over conferences, festivals and special events. All regions are required to establish special organizations to take charge of the publicity and education of public security and emergency management knowledge according to law and, especially, strengthen the emergency management over conferences, festivals and special events. Besides, they need to publicize the knowledge concerning the precaution, avoiding, self-rescue, disaster reduction and all sorts of emergency protection in conferences, festivals and special events. The undertaker of a conference, festival and special event more needs to spare no efforts to carry out the publicity, education and training etc. of knowledge concerning security emergency management of enterprises and personnel involving in the conference, festival and special event, especially provide the publicity, education and interpretation of emergency plans; in this way, this can enhance the participants' crisis awareness and responsibility consciousness so as to form a favorable situation dominated by precaution and followed by the participation by all people. On the basis of continuously strengthening publicity and education, it is also required to form a rapid, timely and efficient information feedback system according to the requirements of conferences, festivals and special events so as to rapidly and timely give feedbacks about any potential safety hazard and the specific condition of an event during a conference, festival and special event to the outside world; therefore, this can attain the goal of more efficient and smooth information transfer, completely eradicate the concealment or false report etc. and practically avoid misreport or false report etc. As for the conference, festival and special event industry in a region, perfecting a feedback system includes the following two aspects: on the one hand, give feedbacks upward; namely, the precaution mechanism can correctly monitor the practical condition of public security and the situation of an emergency, and rapidly and correctly report such conditions to the departments concerned so that the emergency command center for conference, festival and special event can effectively command the public safety protection, maximally reduce and avoid various losses and guarantee that a conference, festival and special event can be carried out smoothly; on the other hand, strengthen external feedbacks; namely, actively and positively report the development situation of related activities and events to the public, form information communication, exchange and interaction with the public, continuously improve measures to prevent the information source for 
an emergency and the most reasonable handling method, thus reasonably deal with an emergency at its initial stage as far as possible and reduce the influence on and losses to a conference, festival and special event.

\section{To comprehensively research the condition on the site of a conference and festival activity}

A conference, festival or special event is one or several actions carried out by people in corresponding conditions, so the security emergency response plan for conferences, festivals and special events mainly involve two factors: human and material. Carefully analyze the situation on the site of a conference, festival or special event to provide a reasonable basis for making a detailed and effective emergency response plan. The main content of a plan covers the two aspects below: on the one hand, classify the safety accidents in conferences, festivals and special events. Based on the two basic factors involved in a security emergency, the safety accidents in conferences, festivals and special events can be divided into three types, which are respectively personal safety accident, material safety accident and comprehensive safety accident. The last safety accident refers to an accident involving both human and materials simultaneously. On the other hand, quickly determine the security emergency grades. Based on the classification of safety accidents on the site of conference, festival and special event, it is required to determine the security emergency response grades accordingly and enter different conditions. Specifically, once a personal casualty accident occurs or probably occur on the site of a conference, festival and special event, immediately enter into the grade-I security emergency state, namely, emergency rescue state. Once congestion occurs or a safety accident is probably incurred from damages of some articles and a hazardous article is brought to the site of a conference, festival and special event, enter into the comprehensive emergency state and then take suitable emergency measures accordingly; this is the grade-II safety emergency. In addition, once there is a doubt of building or equipment damage, which is unlikely to cause a major safety issue, on the site of a conference, festival and special event, enter into the grade-III emergency state; namely, stand by for any need which may arise anytime in the future.

\section{To form a sound and perfect emergency organization and response mechanism}

The construction of conference, festival and special event security system is a process to implement multi-organization and systematic coordination because the single use of the scheme of the venue provider cannot help to absolutely successfully deal with all sorts of safety accidents. The emergency rescue organization for conferences, festivals and special events must be jointly constructed by the commander-in-chief of activity headquarters, higher competent organization of the activity organizer and the emergency command center for activity safety, which have different responsibilities. The commander-in-chief, which is assumed by the top leader of the management organization on the site of conference activity or one appointed by the top leader, mainly takes charge of comprehensively supervising the emergency response work on the site of conference, festival and special event so as to guarantee the effectiveness of accident emergency response plan for the conference, festival and special event; the emergency command center for activity safety is completely under the leadership of emergency rescue leading group and its main work is to organize and coordinate the emergency rescue and treatment on the site, and timely, rapidly give feedbacks about the real-time condition and dynamic changes of rescue on the site of conference, festival and special event to the superior. If an emergency occurs in the conference, festival and special event, the commander-in-chief of conference, festival and special event must go to the site in person and spare no efforts to command the rescue units, venue provider, public security department, fire force and hospital etc. to jointly do rescue work well. Meanwhile, the local people's government of conference, festival and special event shall be comprehensively responsible for a terrorist attack, unlawful seizure, explosive threat and other events endangering the social public security. If necessary, the security emergency organization of conference, festival and special event should actively search for personnel support and information support from the units on the site so as to guarantee the smooth implementation of the emergency response plan. However, in the process, it is required to guarantee the personal safety of such units and their employees with all its strength. The author always thinks that the response to a safety 
accident of conference, festival and special event must always follow the principle of specific response to a specific event so that we can properly adjust, modify, supplement and perfect the emergency response plan for conference, festival and special event in good time. Only in this way, can we minimize the influence of a safety accident to practical reduce people's losses and damages incurred herefrom.

\section{To emphasize the proper implementation of multi-lateral cooperation}

The conference, festival and special event industry is an industry full of crises and frequent occurrence of crises and the related activities organized involve all aspects of urban life, so a sudden public safety accident (if any) fully needs to be handled by multiple departments through concerted action. Firstly, based on all kinds of advanced experience and technologies at home and abroad, the past working experience and the current situation, classify various potential sudden emergencies in the conference, festival and special event, decompose major work tasks and comprehensively clarify the specific responsible units. All members of conferences, festivals and special events, such as the Ministry of State Safety, Public Security Bureau, National Health and Family Planning Commission, fire department and all other departments, should divide their work according to the task, really perform their own respective functions, cooperate with each other closely and establish the strict, efficient, joint and cooperative emergency response mechanism combining protection of major objects and responsibility by specially-assigned personnel. A perfect emergency response mechanism for conferences, festivals and special events mainly consists of the content below: firstly, the emergency command center for conferences, festivals and special events should rapidly start and carry out work, find out, approve and activate the emergency response mechanism of the corresponding grade based on the harmfulness of an event, and implement the emergency organization and commanding of a public safety event; secondly, the security organization of a conference, festival and special event should rapidly enter into the emergency state and respond quickly with instructions; thirdly, establish an information issuing platform for conferences, festival and special events. Based on the activity information management system, establish a platform for centralized issuing of information on public security events so that the platform can rapidly and timely release authoritative information approved officially; fourthly, give full play to the dominant function of a professional rescuer team, form a emergency rescue system and set up a fast all-in-one emergency rescue system; fifthly, do all remedial work well. After a security crisis situation is controlled or removed, the departments concerned should find out the causes for the security event and comprehensively and detailedly analyze the accident; besides, they should also pacify the public and strictly investigate the responsibilities of parties concerned.

\section{Conclusion}

In conclusion, the development of conferences, festivals and special events has injected great energy to China's economic development; besides, the supportive industries around a conference, festival and special event, such as traffic industry and advertising can also obtain great development accordingly. Nowadays, due to the eternally changing international and domestic environments, various safety accidents increasingly become the major potential hazards in major conferences, festivals and special events. How to properly respond largely tests the activity organizer's ability to control a large-scale conference, festival and special event. Therefore, it requires the organizers of conferences, festivals and special events to be highly vigilant all the time, actively take measures and strengthen the construction of conference, festival and special event security system so as to restrain all sorts of hazardous factors as far as possible and avoid the occurrence of an safety accident.

\section{Acknowledgement}

Topic: Research on Security Coordination Management over Festivals and Special Events in Fujian-Taiwan Tourist Islands; topic No.: JAS14473 


\section{References}

[1] Lu Xiao. Planning and management of festivals and special events, Shanghai: Shanghai People's Publishing House, 2006.

[2] Huang Xiang and Lian Jiangong. Tourism festivals and brand construction, Tianjin: Nankai University Press, 2007.

[3] Xie Chaowu and Zheng Xiangmin. Research on security management over exhibitions of hotel industry, Journal of Safety Science and Technology, 2007 (3).

[4] Tong Ruipeng. Analysis on accident risks of conference activities and research on countermeasures for security management, Risk Management, 2009 (6).

[5] Liu Minkun and Fan Peng. Model building of safety management concept in conferences and tourism - taking China-ASEAN Expo for example, Journal of Guangxi University (philosophy and social science edition), 2012 (6).

[6] Xu Yunhong and Zhang Min. Research on the construction of security system for folk festival and conference industry in Hebei province, Journal of Handan Polytechnic College, 2015 (1). 